\title{
Erratum to: Abdominal versus laparoscopic hysterectomies for benign diseases: evaluation of morbidity and mortality among 465,798 cases
}

\author{
Amir Wiser • Christina A. Holcroft • Togas Tulandi • \\ Haim A. Abenhaim
}

Published online: 25 September 2014

(C) Springer-Verlag Berlin Heidelberg 2014

\section{Erratum to: Gynecol Surg \\ DOI 10.1007/s10397-013-0781-9}

\section{On page 117, the "Abstract" should read:}

Hysterectomy is the most common major gynecological surgery performed in women. The aim of this study was to compare major morbidity and mortality between abdominal hysterectomy $(\mathrm{AH})$ and laparoscopic hysterectomy $(\mathrm{LH})$ for benign diseases. We performed a retrospective cohort study using the data from Health Cost and Utilization Project Nationwide Inpatient Sample. Women were admitted for hysterectomy for benign diseases between the years 2002 and 2008. In-hospital morbidities and mortalities were identified using the diagnostic and procedural codes classified according to the International Classification of Disease, Ninth Revision, and Clinical Modification. Logistic regression analysis was used to estimate the relationship between the type of hysterectomy and the development of major morbidity and mortality. Of a total 465,798 cases, 389,189 women $(83.6 \%$ ) underwent $\mathrm{AH}$ and the remainders underwent LH $(76,609,16.4 \%)$. The LH group was younger and more likely to be Caucasian than those who underwent AH. Although major morbidities and mortalities were rare, women who underwent LH were less likely to develop thromboembolic events $(0.69 \%$ vs. $0.84 \%$, odds ratio (OR) 0.85 (0.77-0.93)), require blood transfusions ( $2.4 \%$ vs. $4.7 \%$, OR $0.58(0.55-0.61))$, and sustain bowel perforation $(0.07 \%$ vs. $0.13 \%$, OR $0.56(0.42-0.74))$. The mortality rate was also lower in the LH group $(0.01 \%)$ compared with the AH group (0.03\%, OR $0.48(0.24$ $0.95)$ ). Our conclusion was that for benign diseases, laparoscopic hysterectomy is associated with a lower complication rate than abdominal hysterectomy. When possible, hysterectomy performed for benign diseases should be performed with minimally invasive technique.

On page 119, the last paragraph of the result section should read:

The mode of hysterectomy and the risks of major mortality and morbidity rates are listed in Table 2. Overall, the rates of complications were very low. Even so, the patients who underwent LH had lower morbidity (deep vein thrombosis, pulmonary embolism, and requirement for blood transfusion) as well as less mortality compared to women in the AH group. (The last line was removed).

Table 1 and 2 should read

The online version of the original article can be found at http://dx.doi.org/ 10.1007/s10397-013-0781-9.

\footnotetext{
A. Wiser · C. A. Holcroft · T. Tulandi $(\bowtie) \cdot$ H. A. Abenhaim Department of Obstetrics and Gynecology, McGill University, 687 Pine Avenue West, Montreal, Quebec H3A 1A1, Canada e-mail: togas.tulandi@megill.ca

T. Tulandi Centre for Clinical Epidemiology and Community Studies, Jewish General Hospital, Montreal, Quebec, Canada
} 
Table 1 Baseline characteristics of 465,798 patients who underwent laparoscopic vs. abdominal hysterectomies for benign disease
${ }^{\text {a }}$ Missing comorbidity data in 5,324 records

\begin{tabular}{|c|c|c|}
\hline characteristic & $\begin{array}{l}\text { Open TAH } \\
(\mathrm{n}=389,189)\end{array}$ & $\begin{array}{l}\text { Laparoscopy } \\
(\mathrm{n}=76,609)\end{array}$ \\
\hline \multicolumn{3}{|l|}{ Age } \\
\hline$<35$ & $29,792(8 \%)$ & $8,107(11 \%)$ \\
\hline $35-39$ & $56,658(15 \%)$ & $12,295(16 \%)$ \\
\hline $40-44$ & $106,817(27 \%)$ & $19,256(25 \%)$ \\
\hline $45-49$ & $110,282(28 \%)$ & $20,301(27 \%)$ \\
\hline$\geq 50$ & $85,399(22 \%)$ & $16,606(22 \%)$ \\
\hline \multicolumn{3}{|l|}{ Race } \\
\hline Caucasian & $170,001(44 \%)$ & $43,174(56 \%)$ \\
\hline African-American & $62,291(16 \%)$ & $5,963(8 \%)$ \\
\hline Hispanic & $31,295(8 \%)$ & $4,569(6 \%)$ \\
\hline Other & $17,100(4 \%)$ & $2,627(3 \%)$ \\
\hline Unknown & $108,492(28 \%)$ & $20,276(26 \%)$ \\
\hline \multicolumn{3}{|l|}{ Median income (US\$) } \\
\hline$<35,000$ & $95,202(24 \%)$ & $15,921(21 \%)$ \\
\hline $35,000-44,999$ & $97,967(25 \%)$ & $19,278(25 \%)$ \\
\hline$\geq 45,000$ & $187,998(48 \%)$ & $39,707(52 \%)$ \\
\hline \multicolumn{3}{|l|}{ Insurance type } \\
\hline Medicare & $21,399(6 \%)$ & $3,668(5 \%)$ \\
\hline Medicaid & $34,267(9 \%)$ & $5,207(7 \%)$ \\
\hline Private & $304,072(78 \%)$ & $63,140(82 \%)$ \\
\hline Other & $28,726(7 \%)$ & $4,456(6 \%)$ \\
\hline \multicolumn{3}{|l|}{ Hospital } \\
\hline Rural & $50,239(13 \%)$ & $10,455(14 \%)$ \\
\hline Urban, non-teaching & $177,299(46 \%)$ & $36,546(48 \%)$ \\
\hline Urban, teaching & $161,324(41 \%)$ & $29,570(39 \%)$ \\
\hline \multicolumn{3}{|l|}{ Admission } \\
\hline Elective & $338,668(87 \%)$ & $68361(89 \%)$ \\
\hline Non-elective & $48453(12 \%)$ & $7,898(10 \%)$ \\
\hline \multicolumn{3}{|l|}{ Comorbidities $^{a}$} \\
\hline Congestive heart failure & $1,539(0.4 \%)$ & $125(0.2 \%)$ \\
\hline Chronic pulmonary disease & $26,585(7 \%)$ & $5,100(7 \%)$ \\
\hline Diabetes (complicated or uncomplicated) & $21,998(6 \%)$ & $3,159(4 \%)$ \\
\hline Hypertension (complicated or uncomplicated & $79,065(20 \%)$ & $12,028(16 \%)$ \\
\hline Lymphoma & $265(0.07 \%)$ & $40(0.05 \%)$ \\
\hline Peripheral vascular disorder & $499(0.1 \%)$ & $68(0.09 \%)$ \\
\hline
\end{tabular}


Table 2 Effect of hysterectomy approach on the risk of major morbidities and mortality

\begin{tabular}{|c|c|c|c|c|}
\hline Outcome & Abdominal hysterectomy & Laparoscopic hysterectomy & Adjusted OR (95 \% CI) & $\mathrm{P}$ value \\
\hline DVT & $2,879(0.74 \%)$ & $502(0.66 \%)$ & $0.91(0.82,0.99)$ & 0.04 \\
\hline $\mathrm{PE}$ & $3,099(0.80 \%)$ & $522(0.68 \%)$ & $0.88(0.80,0.96)$ & 0.006 \\
\hline DVT or PE & $3,281(0.84 \%)$ & $529(0.69 \%)$ & $0.85(0.77,0.93)$ & 0.0004 \\
\hline Blood transfusion & $18,124(4.7 \%)$ & $1,805(2.4 \%)$ & $0.58(0.55,0.61)$ & $<0.0001$ \\
\hline Bowel perforation & $490(0.13 \%)$ & $52(0.07 \%)$ & $0.56(0.42,0.74)$ & $<0.0001$ \\
\hline Bladder injury & $17(<0.01 \%)$ & $0(0 \%)$ & N/A & N/A \\
\hline Acute myocardial infarction & $133(0.03 \%)$ & $13(0.02 \%)$ & $0.69(0.39,1.2)$ & 0.2 \\
\hline Length of stay $\geq 6$ days & $15,917(4.1 \%)$ & $804(1.1 \%)$ & $0.29(0.27,0.31)$ & $<0.0001$ \\
\hline Death & $123(0.03 \%)$ & $9(0.01 \%)$ & $0.48(0.24,0.95)$ & 0.036 \\
\hline
\end{tabular}

DVT deep vein thrombosis, PE pulmonary embolism, MI myocardial infarction 